\title{
An Additive Basis for the Chow Ring of $\overline{\mathcal{M}}_{0,2}\left(\mathbb{P}^{r}, 2\right)$
}

Jonathan A. COX

Department of Mathematical Sciences, SUNY Fredonia, Fredonia, New York 14063, USA

E-mail: Jonathan.Cox@fredonia.edu

URL: http://www.fredonia.edu/faculty/math/JonathanCox/

Received July 03, 2007, in final form August 28, 2007; Published online August 31, 2007

Original article is available at http://www.emis.de/journals/SIGMA/2007/085/

\begin{abstract}
We begin a study of the intersection theory of the moduli spaces of degree two stable maps from two-pointed rational curves to arbitrary-dimensional projective space. First we compute the Betti numbers of these spaces using Serre polynomial and equivariant Serre polynomial methods developed by E. Getzler and R. Pandharipande. Then, via the excision sequence, we compute an additive basis for their Chow rings in terms of Chow rings of nonlinear Grassmannians, which have been described by Pandharipande. The ring structure of one of these Chow rings is addressed in a sequel to this paper.
\end{abstract}

Key words: moduli space of stable maps; Chow ring; Betti numbers

2000 Mathematics Subject Classification: 14C15; 14D22

\section{Introduction}

Let $\overline{\mathcal{M}}_{g, n}\left(\mathbb{P}^{r}, d\right)$ be the moduli space of stable maps from $n$-pointed, genus $g$ curves to $\mathbb{P}^{r}$ of degree $d$. In this article we will begin a study of the intersection theory of the moduli spaces $\overline{\mathcal{M}}_{0,2}\left(\mathbb{P}^{r}, 2\right)$.

Moduli spaces of stable maps have proven useful in studying both superstring theory and enumerative geometry. Developing a solid mathematical foundation for computation of certain numbers in string theory was the primary motivation behind the introduction of moduli spaces of stable maps in [14]. Examples include the instanton numbers, which intuitively count the number of holomorphic instantons on a space $X$ (nonconstant holomorphic maps from Riemann surfaces to $X$ ). Instanton numbers are calculated using other values called Gromov-Witten invariants. Naively, Gromov-Witten invariants should count the number of curves of a certain homology class and genus which pass through certain subvarieties of the target space. More specifically, let $X$ be a projective manifold, $\beta \in H_{2}(X), g$ and $n$ nonnegative integers. Let $\gamma_{1}, \ldots, \gamma_{n} \in H^{*}(X)$ be cohomology classes such that there exist subvarieties $Z_{1}, \ldots, Z_{n}$ with $Z_{i}$ representing the Poincaré dual of $\gamma_{i}$. Then the Gromov-Witten invariant $\left\langle\gamma_{1}, \ldots, \gamma_{n}\right\rangle_{g, \beta}$ should count the number of genus $g$ curves of class $\beta$ that intersect all of the $Z_{i}$. Also of interest are gravitational correlators, which generalize Gromov-Witten invariants. Gravitational correlators are defined and computed mathematically as intersection numbers on the moduli space of stable maps. See [3, Chapter 10] for a rigorous definition and the sequel [4] for some computations that flow from the results of this paper.

In the dozen years since Kontsevich introduced the concept in [14] and [13], the moduli space of stable maps has been exploited to solve a plethora of enumerative problems for curves. As a rule, these results were derived without a complete description of the Chow rings involved. Instead, the requisite intersection numbers were calculated somewhat indirectly, most often using the method of localization. (An overview of localization is given in [3, Chapter 9].) Such a complete description is the key step in giving another, more direct, computation of these enumerative numbers and possibly many others. Since a presentation for a ring gives an easy 
way to compute all products in the ring, giving presentations for the Chow rings of moduli spaces of stable maps is the clear path toward attaining a full and direct knowledge of their intersection theory. As a consequence, this also helps give a new and more direct way of determining values of instanton numbers, Gromov-Witten invariants, and gravitational correlators.

Until recently, presentations for Chow rings of moduli spaces of stable maps were known only in a few special cases. Most of these had projective space as the target of the stable maps, and in this case the moduli space $\overline{\mathcal{M}}_{g, n}\left(\mathbb{P}^{r}, d\right)$ depends on four nonnegative integer parameters: the genus $g$ of the curves, the number $n$ of marked points on the curves, the dimension $r$ of the target projective space, and the degree $d$ of the stable maps. Most impressive was the presentation of $A^{*}\left(\overline{\mathcal{M}}_{0,1}\left(\mathbb{P}^{r}, d\right)\right)$ for arbitrary $d$ and $r$ described by Mustata and Mustata in [17]. Behrend and O'Halloran gave a presentation for $A^{*}\left(\overline{\mathcal{M}}_{0,0}\left(\mathbb{P}^{r}, 2\right)\right)$ and conjectured a presentation for $A^{*}\left(\overline{\mathcal{M}}_{0,0}\left(\mathbb{P}^{r}, 3\right)\right)$ in [2]. Also of relevance, Oprea more recently described a system of tautological subrings of the cohomology (and hence Chow) rings in the genus zero case and showed that, if the target $X$ is an SL flag variety, then all rational cohomology classes on $\overline{\mathcal{M}}_{0, n}(X, \beta)$ are tautological. This gave, at least in principle, a set of generators for any such Chow ring, namely its tautological classes. He furthermore described an additive generating set for the cohomology ring of any genus zero moduli space (with target a projective algebraic variety). Finally, he speculated that all relations between the tautological generators are consequences of the topological recursion relations. These developments were substantial steps toward describing presentations for the Chow rings of moduli spaces of stable maps in much more general cases. See [19] and [20] for more details. More basic examples include $A^{*}\left(\overline{\mathcal{M}}_{0, n}\left(\mathbb{P}^{r}, 0\right)\right) \simeq A^{*}\left(\mathbb{P}^{r}\right) \times A^{*}\left(\bar{M}_{0, n}\right)$, where $\bar{M}_{0, n}$ is the moduli space of stable curves. This case reduces to finding presentations for the rings $A^{*}\left(\bar{M}_{0, n}\right)$, and Keel did so in [11]. Also, $\overline{\mathcal{M}}_{0,0}\left(\mathbb{P}^{r}, 1\right)$ is isomorphic to $\mathbb{G}(1, r)$, the Grassmannian of lines in projective space, and $\overline{\mathcal{M}}_{0,1}\left(\mathbb{P}^{r}, 1\right)$ is isomorphic to $\mathbb{F}(0,1 ; r)$, the flag variety of pointed lines in projective space. The spaces $\overline{\mathcal{M}}_{0, n}\left(\mathbb{P}^{1}, 1\right)$ are Fulton-MacPherson compactifications of configuration spaces of $\mathbb{P}^{1}$. Presentations for their Chow rings were given by Fulton and MacPherson in [7]. Detailed descriptions of Chow rings of spaces $\overline{\mathcal{M}}_{g, n}\left(\mathbb{P}^{r}, d\right)$, with $g>0$, are almost nonexistent (although some progress is now being made for $g=1$ ). Additional complications arise in this case.

This was the state of affairs up to the posting of this article, which lays the foundation for computing presentations of the Chow rings of the spaces $\overline{\mathcal{M}}_{0,2}\left(\mathbb{P}^{r}, 2\right)$. This computation is completed for the case $r=1$ in [4], the sequel to this article, where we obtain the presentation

$$
A^{*}\left(\overline{\mathcal{M}}_{0,2}\left(\mathbb{P}^{1}, 2\right)\right) \simeq \frac{\mathbb{Q}\left[D_{0}, D_{1}, D_{2}, H_{1}, H_{2}, \psi_{1}, \psi_{2}\right]}{\left(\begin{array}{c}
H_{1}^{2}, H_{2}^{2}, D_{0} \psi_{1}, D_{0} \psi_{2}, D_{2}-\psi_{1}-\psi_{2}, \psi_{1}-\frac{1}{4} D_{1}-\frac{1}{4} D_{2}-D_{0}+H_{1}, \\
\psi_{2}-\frac{1}{4} D_{1}-\frac{1}{4} D_{2}-D_{0}+H_{2},\left(D_{1}+D_{2}\right)^{3}, D_{1} \psi_{1} \psi_{2}
\end{array}\right)}
$$

This gave the first known presentation for a Chow ring of a moduli space of stable maps of degree greater than one with more than one marked point. The sequel also employs the presentation to give a new computation of the genus zero, degree two, two-pointed gravitational correlators of $\mathbb{P}^{1}$. Algorithms for computing theses values have previously been developed; see [15] and [3], for example.

Knowing the Betti numbers of $\overline{\mathcal{M}}_{0,2}\left(\mathbb{P}^{r}, 2\right)$ is the important first step in our computation, since it will give us a good idea of how many generators and relations to expect in each degree. We accomplish this in Section 2 by using the equivariant Serre polynomial method of Getzler and Pandharipande. I owe much gratitude to Getzler and Pandharipande for supplying a copy of their unpublished preprint [9], which provided the inspiration for Section 2. In Section 3, we use the presentations of the Chow rings for the moduli spaces $\mathcal{M}_{0,0}\left(\mathbb{P}^{r}, d\right)$ from [21] together with excision to determine a generating set for the Chow ring $A^{*}\left(\overline{\mathcal{M}}_{0,2}\left(\mathbb{P}^{r}, 2\right)\right)$. Comparing the dimension of each graded piece with the Betti numbers from Section 2, we conclude that this generating set is actually an additive basis. 
Since the completion of this article, additional results have been announced. Getzler and Pandharipande have substantially reworked the material from and completed the computations begun in their preprint, giving Betti numbers for all the spaces $\overline{\mathcal{M}}_{0, n}\left(\mathbb{P}^{r}, d\right)$ in [10]. However, they do this via an indirect method, using a generalization of the Legendre transform, and the result is an algorithm for computing the Betti numbers rather than a closed formula for them. The approach taken here is more straightforward and sheds more light on the geometry involved. Finally, Mustata and Mustata extended their results for one-pointed spaces to supply presentations for the Chow rings of all spaces $\overline{\mathcal{M}}_{0, n}\left(\mathbb{P}^{r}, d\right)$ in [18]. Again, the methods of the present paper are simpler, and the presentation obtained in the sequel is more explicit.

\subsection{Conventions and preliminary comments}

We will work over the field $\mathbb{C}$ of complex numbers. Let $\underline{n}=\mathbb{N} \cap[1, n]$ be the initial segment consisting of the first $n$ natural numbers.

The moduli stack $\overline{\mathcal{M}}_{g, n}\left(\mathbb{P}^{r}, d\right)$ captures all the data of the moduli problem for stable maps, while the moduli scheme $\bar{M}_{g, n}\left(\mathbb{P}^{r}, d\right)$ loses some information, including that of automorphisms of families. Since retaining all of this data leads to a more beautiful, powerful, and complete theory, we will work with the stack incarnations of the moduli spaces rather than the coarse moduli schemes.

Let $H^{*}(F)$ denote the rational de Rham cohomology ring of a Deligne-Mumford stack $F$.

Proposition 1 (Homology isomorphism). Let $X$ be a flag variety. Then there is a canonical ring isomorphism

$$
A^{*}\left(\overline{\mathcal{M}}_{0, n}(X, \beta)\right) \rightarrow H^{*}\left(\overline{\mathcal{M}}_{0, n}(X, \beta)\right) .
$$

See [19] for a proof. Following [11], we call any scheme or stack $Y$ an $H I$ scheme or $H I$ stack if the canonical map $A^{*}(Y) \rightarrow H^{*}(Y)$ is an isomorphism. In particular, by Proposition 1 $Y=\overline{\mathcal{M}}_{0, n}\left(\mathbb{P}^{r}, d\right)$ is an HI stack since $\mathbb{P}^{r}$ is a flag variety. This allows us to switch freely between cohomology and Chow rings. We should note that the isomorphism doubles degrees. The degree of a $k$-cycle in $A^{k}\left(\overline{\mathcal{M}}_{0, n}(X, \beta)\right)$, called the algebraic degree, is half the degree of its image in $H^{2 k}\left(\overline{\mathcal{M}}_{0, n}(X, \beta)\right)$.

\section{The Betti numbers of $\overline{\mathcal{M}}_{0,2}\left(\mathbb{P}^{r}, 2\right)$}

\subsection{Serre polynomials and the Poincaré polynomial of $\overline{\mathcal{M}}_{0,2}\left(\mathbb{P}^{r}, 2\right)$}

This section owes much to Getzler and Pandharipande, who provide the framework for computing the Betti numbers of all the spaces $\overline{\mathcal{M}}_{0, n}\left(\mathbb{P}^{r}, d\right)$ in [9]. However, we will take the definitions and basic results from other sources, and prove their theorem in the special case that we need. We will compute a formula for the Poincaré polynomials of the moduli spaces $\overline{\mathcal{M}}_{0,2}\left(\mathbb{P}^{r}, 2\right)$ using what are called Serre polynomials in [9] and Serre characteristics in [10]. (These polynomials are also known as virtual Poincaré polynomials or E-polynomials.) Serre polynomials are defined for varieties over $\mathbb{C}$ via the mixed Hodge theory of Deligne ([6]). Serre conjectured the existence of polynomials satisfying the key properties given below. A formula was later given by Danilov and Khovanskiı̌ in [5]. If $(V, F, W)$ is a mixed Hodge structure over $\mathbb{C}$, set

$$
V^{p, q}=F^{p} \operatorname{gr}_{p+q}^{W} V \cap \bar{F}^{q} \operatorname{gr}_{p+q}^{W} V
$$

and let $\mathcal{X}(V)$ be the Euler characteristic of $V$ as a graded vector space. Then

$$
\operatorname{Serre}(X)=\sum_{p, q=0}^{\infty} u^{p} v^{q} \mathcal{X}\left(H_{c}^{\bullet}(X, \mathbb{C})^{p, q}\right) .
$$


If $X$ is a smooth projective variety, then the Serre polynomial of $X$ is just its Hodge polynomial:

$$
\operatorname{Serre}(X)=\sum_{p, q=0}^{\infty}(-u)^{p}(-v)^{q} \operatorname{dim} H^{p, q}(X, \mathbb{C}) .
$$

If $X$ further satisfies $H^{p, q}(X, \mathbb{C})=0$ for $p \neq q$, then we can substitute a new variable $q=u v$ for $u$ and $v$. In this case, the coefficients of the Serre polynomial of $X$ give its Betti numbers, so that $\operatorname{Serre}(X)$ is the Poincaré polynomial of $X$.

We will use two additional key properties of Serre polynomials. The first gives a compatibility with decomposition: If $Z$ is a closed subvariety of $X$, then $\operatorname{Serre}(X)=\operatorname{Serre}(X \backslash Z)+\operatorname{Serre}(Z)$. Second, it respects products: $\operatorname{Serre}(X \times Y)=\operatorname{Serre}(X) \operatorname{Serre}(Y)$. (The latter is actually a consequence of the previous properties.) It follows from these two properties that the Serre polynomial of a fiber space is the product of the Serre polynomials of the base and the fiber. The definition and properties above come from [8]. We also use the following consequence of the EilenbergMoore spectral sequence, which is essentially Corollary 4.4 in [22].

Proposition 2. Let $Y \rightarrow B$ be a fiber space with $B$ simply connected, and let $X \rightarrow B$ be continuous. If $H^{*}(Y)$ is a free $H^{*}(B)$-module, then

$$
H^{*}\left(X \times_{B} Y\right) \simeq H^{*}(X) \otimes_{H^{*}(B)} H^{*}(Y)
$$

as an algebra.

Since we deal exclusively with cases where the isomorphism (1) holds, there is never any torsion in the cohomology. Thus we have the following.

Corollary 1. Let $X$ and $Y$ be varieties over a simply connected base B, and suppose either $X$ or $Y$ is locally trivial over $B$. Then

$$
\operatorname{Serre}\left(X \times_{B} Y\right)=\frac{\operatorname{Serre}(X) \operatorname{Serre}(Y)}{\operatorname{Serre}(B)} .
$$

We will sometimes use the notation $Y / B$ for the fiber of a fiber space $Y \rightarrow B$.

To extend this setup to Deligne-Mumford stacks, where automorphism groups can be nontrivial (but still finite), equivariant Serre polynomials are needed. Let $G$ be a finite group acting on a variety $X$. The idea is this: The action of $G$ on $X$ induces an action on its cohomology (preserving the mixed Hodge structure), which in turn gives a representation of $G$ on each (bi)graded piece of the cohomology. The cohomology of the quotient variety $X / G$, and hence of the quotient stack $[X / G]$, is the part of the cohomology of $X$ which is fixed by the $G$-action, i.e., in each degree the subspace on which the representation is trivial.

Our definition comes from [8]. The equivariant Serre polynomial $\operatorname{Serre}(X, G)$ of $X$ is given by the formula

$$
\text { Serre }_{g}(X)=\sum_{p, q=0}^{\infty} u^{p} v^{q} \sum_{i}(-1)^{i} \operatorname{Tr}\left(g \mid\left(H_{c}^{i}(X, \mathbb{C})\right)^{p, q}\right) .
$$

for each element $g \in G$. We can also describe the equivariant Serre polynomial more compactly with the formula

$$
\operatorname{Serre}(X, G)=\sum_{p, q=0}^{\infty} u^{p} v^{q} \sum_{i}(-1)^{i}\left[H_{c}^{i}(X, \mathbb{C})^{p, q}\right],
$$

taken from [9]. In the case $G=S_{n}$, we write $\operatorname{Serre}_{n}(X)$ for $\operatorname{Serre}\left(X, S_{n}\right)$. A $G$-equivariant Serre polynomial takes values in $R(G)[u, v]$, where $R(G)$ is the virtual representation ring of 
$G$. The augmentation morphism $\epsilon: R(G) \rightarrow \mathbb{Z}$, which extracts the coefficient of the trivial representation $\mathbb{1}$ from an element of $R(G)$, extends to an augmentation morphism $R(G)[u, v] \rightarrow$ $\mathbb{Z}[u, v]$. If $G$ acts on a quasi-projective variety $X$, the Serre polynomial of the quotient stack $[X / G]$ is the augmentation of the equivariant Serre polynomial of $X$.

Every virtual representation $\operatorname{ring} R(G)$ has the extra structure of a $\lambda$-ring. See [12] for the definitions and basic properties of $\lambda$-rings and pre- $\lambda$-rings. Here we just briefly state the most relevant facts.

Let $V$ be a $G$-module. Then $\lambda_{i}(V)$ is the $i$ 'th exterior power $\Lambda^{i} V$ of $V$, where we define $g \in G$ to act by $g\left(v_{1} \wedge \cdots \wedge v_{i}\right)=g v_{1} \wedge \cdots \wedge g v_{i}$. Define $\lambda_{0}(V)$ to be the trivial one-dimensional representation. (One can similarly define a $G$-module structure on the $i$ 'th symmetric power $S^{i} V$.) Knutson proves in [12, Chapter II] that these exterior power operations give $R(G)$ the structure of a $\lambda$-ring for any finite group $G$. Addition is given by $[V]+[W]=[V \oplus W]$, and the product is $[V] \cdot[W]=[V \otimes W]$, both with the naturally induced actions.

Knutson also shows that $\mathbb{Z}$ is a $\lambda$-ring with $\lambda$-operations given via $\lambda_{t}(m)=(1+t)^{m}$, where by definition $\lambda_{t}(m)=\sum \lambda_{i}(m) t^{i}$. For $m, n \geq 0$, this gives $\lambda_{n}(m)=\left(\begin{array}{c}m \\ n\end{array}\right)$. Finally, he shows that if $R$ is a $\lambda$-ring, then there is a unique structure of $\lambda$-ring on $R[x]$ under which $\lambda_{k}\left(r X^{n}\right)=\lambda_{k}(r) X^{n k}$ for $n, k \in \mathbb{N} \cup\{0\}$ and $r \in R$. This gives a $\lambda$-ring structure on $\mathbb{Z}[q]$. The augmentation morphism $\epsilon: R(G) \rightarrow \mathbb{Z}$ is a map of $\lambda$-rings; it commutes with the $\lambda$-operations.

We will use the following facts about Serre polynomials and equivariant Serre polynomials. For $n \in \mathbb{N}$, let $[n]=\frac{q^{n}-1}{q-1}$. Then $[n+1]$ is the Serre polynomial of $\mathbb{P}^{n}$, as is clear from the presentation for its Chow ring. Getzler and Pandharipande prove that the Serre polynomial of the Grassmannian $G(k, n)$ of $k$-planes in $\mathbb{C}^{n}$ is the $q$-binomial coefficient

$$
\left[\begin{array}{l}
n \\
k
\end{array}\right]=\frac{[n] !}{[k] ![n-k] !}
$$

where $[n] !=[n][n-1] \cdots[2][1]$. We will prove this formula in the special case $k=2$.

Lemma 1. The Serre polynomial of $G(2, n)$ is $\left[\begin{array}{l}n \\ 2\end{array}\right]$.

Proof. We can work with the Grassmannian $\mathbb{G}(1, n-1)$ of lines in $\mathbb{P}^{n-1}$ since $G(2, n) \simeq$ $\mathbb{G}(1, n-1)$. The universal $\mathbb{P}^{1}$-bundle over $\mathbb{G}(1, n-1)$ is isomorphic to $\mathbb{F}(0,1 ; n-1)$, the flag variety of pairs $(p, \ell)$ of a point $p$ and a line $\ell$ in $\mathbb{P}^{n-1}$ with $p \in \ell$. On the other hand, there is a projection $\mathbb{F}(0,1 ; n-1) \rightarrow \mathbb{P}^{n-1}$ taking $(p, \ell)$ to $p$. Its fiber over a point $p$ is $\{\ell \mid p \in \ell\}$, which is isomorphic to $\mathbb{P}^{n-2}$. (To see this isomorphism, fix a hyperplane $H \subset \mathbb{P}^{n-1}$ not containing $p$ and map each line to its intersection with $H$.) It follows that $\operatorname{Serre}(\mathbb{F}(0,1 ; n-1))=[n][n-1]$. Since $\operatorname{Serre}(\mathbb{F}(0,1 ; n-1))=\operatorname{Serre}(\mathbb{G}(1, n-1))[2]$ also, we are able to conclude that Serre $(\mathbb{G}(1, n-1))=$ $[n][n-1] /[2]$.

Next, since PGL(2) is the complement of a quadric surface in $\mathbb{P}^{3}$, Serre $(\operatorname{PGL}(2))=[4]-[2]^{2}=$ $q^{3}-q$.

In addition to the $\lambda$-operations, every $\lambda$-ring $R$ has $\sigma$-operations as well. These can be defined in terms of the $\lambda$-operations by $\sigma_{k}(x)=(-1)^{k} \lambda_{k}(-x)$. Routine checking shows that the $\sigma$-operations also give $R$ the structure of a pre- $\lambda$-ring. Here we simply note the following formulas for the $\lambda$-ring $\mathbb{Z}[q]$

$$
\sigma_{k}([n])=\left[\begin{array}{c}
n+k-1 \\
k
\end{array}\right] \quad \text { and } \quad \lambda_{k}([n])=q^{\left(\begin{array}{c}
k \\
2
\end{array}\right)}\left[\begin{array}{l}
n \\
k
\end{array}\right] .
$$

Proofs of these formulas can be found in [16, Section I.2]. Next we explain why these formulas are relevant. Let $\epsilon$ be the sign representation of $S_{n}$. Note the identity $\epsilon^{2}=\mathbb{1}$. We will prove the following claim from [9]. 
Lemma 2. If $X$ is a smooth variety and $S_{2}$ acts on $X^{2}$ by switching the factors, then

$$
\operatorname{Serre}_{2}\left(X^{2}\right)=\sigma_{2}(\operatorname{Serre}(X)) \mathbb{1}+\lambda_{2}(\operatorname{Serre}(X)) \epsilon .
$$

Proof. Let $V$ be a vector space. Now $V \otimes V=S^{2} V \oplus \Lambda^{2} V$ as $S_{2}$-modules, with $S_{2}$ acting by switching the factors of $V \otimes V$, trivially on $S^{2} V$, and by sign on $\Lambda^{2} V$. If 0 is the zero representation, certainly $\lambda_{i}(0)=0$ for $i>0$. We use this fact and the properties of $\lambda$-rings to obtain

$$
\begin{aligned}
0 & =\lambda_{2}([V]-[V])=\mathbb{1} \cdot \lambda_{2}(-[V])+[V] \cdot(-[V])+\lambda_{2}[V] \cdot \mathbb{1} \\
& =\lambda_{2}(-[V])-\left[S^{2} V\right]-\left[\Lambda^{2} V\right]+\lambda_{2}[V] .
\end{aligned}
$$

Since $\sigma_{2}[V]=\lambda_{2}(-[V])$, this implies $\sigma_{2}[V]=\left[S^{2} V\right]$. Since $X$ is smooth, $H^{*}\left(X^{2}\right)=H^{*}(X) \otimes$ $H^{*}(X)$, with the action of $S_{2}$ switching the factors. Applying the above with $V=H^{*}(X)$ gives $\left[H^{*}\left(X^{2}\right)\right]=\sigma_{2}\left[H^{*}(X)\right]+\lambda_{2}\left[H^{*}(X)\right]$. Breaking this down by (cohomological) degree, we have $\left[H^{i}\left(X^{2}\right)\right] q^{i}=\left[\sigma_{2}\left[H^{*}(X)\right]\right]_{i} q^{i}+\left[\lambda_{2}\left[H^{*}(X)\right]\right]_{i} q^{i}$. We need to show that $\left[H^{i}\left(X^{2}\right)\right] q^{i}=$ $\left[\sigma_{2}(\operatorname{Serre}(X))\right]_{i} \mathbb{1}+\left[\lambda_{2}(\operatorname{Serre}(X))\right]_{i} \epsilon$. We will show the equality of the first summands of each expression; showing equality of the terms involving $\lambda_{2}$ is easier. First, by induction the identity $\lambda_{2}(-m)=\lambda_{2}(m+1)$ holds. Second, note that any pre- $\lambda$-operation $\lambda_{2}$ acts on sums by $\lambda_{2}\left(\sum_{i} x_{i}\right)=\sum_{i} \lambda_{2}\left(x_{i}\right)+\sum_{i<j} x_{i} x_{j}$. Third, note that vector spaces in the following computation live in the graded algebra $H^{*}(X) \otimes H^{*}(X)$, and we will apply the usual rules for grading in a tensor product. Finally, all of the representations below are trivial. We find

$$
\begin{aligned}
& {\left[\sigma_{2}\left[H^{*}(X)\right]\right]_{i} q^{i}=\left[\sigma_{2}\left[\sum_{j} H^{j}(X)\right]\right]_{i} q^{i}=\left[\sum_{j}\left[S^{2} H^{j}(X)\right]+\sum_{j<k}\left[H^{j}(X) \otimes H^{k}(X)\right]\right]_{i} q^{i}} \\
& = \begin{cases}\left(\left[S^{2} H^{i / 2}(X)\right]+\sum_{\substack{j+k=i \\
j<k}}\left[H^{j}(X) \otimes H^{k}(X)\right]\right) q^{i} & \text { if } i \text { is even, } \\
\left(\sum_{\substack{j+k=i \\
j<k}}\left[H^{j}(X) \otimes H^{k}(X)\right]\right) q^{i} & \text { if } i \text { is odd, }\end{cases} \\
& = \begin{cases}\left(\left(\begin{array}{c}
h^{i / 2}(X)+1 \\
2
\end{array}\right) \mathbb{1}+\sum_{\substack{j+k=i \\
j<k}} h^{j}(X) h^{k}(X) \mathbb{1}\right) q^{i} & \text { if } i \text { is even, } \\
\left(\sum_{\substack{j+k=i \\
j<k}} h^{j}(X) h^{k}(X) \mathbb{1}\right) q^{i} & \text { if } i \text { is odd. }\end{cases}
\end{aligned}
$$

On the other hand,

$$
\begin{aligned}
& {\left[\sigma_{2}(\operatorname{Serre}(X))\right]_{i} \mathbb{1}=\left[\lambda_{2}\left(-\sum h^{j}(X) q^{j}\right)\right]_{i} \mathbb{1}} \\
& =\left[\sum \lambda_{2}\left(-h^{j}(X)\right) q^{2 j}+\sum_{j<k} h^{j}(X) h^{k}(X) q^{j+k}\right]_{i} \mathbb{1} \\
& = \begin{cases}\left(\left(\begin{array}{c}
h^{i / 2}(X)+1 \\
2
\end{array}\right) q^{i}+\sum_{\substack{j+k=i \\
j<k}} h^{j}(X) h^{k}(X) q^{i}\right) \mathbb{1} & \text { if } i \text { is even } \\
\left(\sum_{\substack{j+k=i \\
j<k}} h^{j}(X) h^{k}(X) q^{i}\right) \mathbb{1} & \text { if } i \text { is odd. }\end{cases}
\end{aligned}
$$


As a corollary, the ordinary Serre polynomial of $\left[X^{2} / S_{2}\right]$ is $\sigma_{2}(\operatorname{Serre}(X))$.

The following proposition gives a key fact used in our computations. Notice that it refers to the locus $\mathcal{M}_{0,0}\left(\mathbb{P}^{r}, d\right)$ ) of stable maps with smooth domain curve, which is a proper (dense) subset of the compactified moduli space $\overline{\mathcal{M}}_{0,0}\left(\mathbb{P}^{r}, d\right)$.

Proposition 3. If $d>0$, $\operatorname{Serre}\left(\mathcal{M}_{0,0}\left(\mathbb{P}^{r}, d\right)\right)=q^{(d-1)(r+1)}\left[\begin{array}{c}r+1 \\ 2\end{array}\right]$.

This follows from Pandharipande's proof in [21] that the Chow ring of the nonlinear Grassmannian $M_{\mathbb{P}^{k}}\left(\mathbb{P}^{r}, d\right)$ is isomorphic to the Chow ring of the ordinary Grassmannian $\mathbb{G}(k, r)$. If $k=1$, the nonlinear Grassmannian is $\mathcal{M}_{0,0}\left(\mathbb{P}^{r}, d\right)$. (The Serre polynomial grades by dimension rather than codimension. This is why the shifting factor $q^{(d-1)(r+1)}$ appears.)

Recall that $\mathcal{M}_{0, n}\left(\mathbb{P}^{r}, 0\right) \simeq M_{0, n} \times \mathbb{P}^{r}$, so that the Serre polynomials of these spaces are easy to compute.

Finally, let $F(X, n)$ be the configuration space of $n$ distinct labeled points in a nonsingular variety $X$. Fulton and MacPherson show in [7] that

$$
\operatorname{Serre}(F(X, n))=\prod_{i=0}^{n-1}(\operatorname{Serre}(X)-i) .
$$

In order to compute the Serre polynomial of a moduli space of stable maps, we can stratify it according to the degeneration types of the maps and compute the Serre polynomial of each stratum separately. The degeneration types of maps in $\overline{\mathcal{M}}_{0, n}\left(\mathbb{P}^{r}, d\right)$ are in $1-1$ correspondence with stable $(n, d)$-trees via taking the dual graph of a stable map. These concepts were defined and developed by Behrend and Manin in [1].

We are now ready to compute the Poincaré polynomials of some moduli spaces of stable maps.

Proposition 4. The Poincaré polynomial of $\overline{\mathcal{M}}_{0,2}\left(\mathbb{P}^{r}, 2\right)$ is

$$
\operatorname{Serre}\left(\overline{\mathcal{M}}_{0,2}\left(\mathbb{P}^{r}, 2\right)\right)=\left(\sum_{i=0}^{r} q^{i}\right)\left(\sum_{i=0}^{r-1} q^{i}\right)\left(\sum_{i=0}^{r+2} q^{i}+2 \sum_{i=1}^{r+1} q^{i}+2 \sum_{i=2}^{r} q^{i}\right)
$$

and the Euler characteristic of $\overline{\mathcal{M}}_{0,2}\left(\mathbb{P}^{r}, 2\right)$ is $r(r+1)(5 r+3)$.

Proof. We begin by stratifying $\overline{\mathcal{M}}_{0,2}\left(\mathbb{P}^{r}, 2\right)$ according to the degeneration type of the stable maps. Since the strata are locally closed, the compatibility of Serre polynomials with decomposition allows us to compute the Serre polynomial of each stratum separately and add up the results to obtain Serre $\left(\overline{\mathcal{M}}_{0,2}\left(\mathbb{P}^{r}, 2\right)\right)$.

Each stratum is isomorphic to a finite group quotient of a fiber product of moduli spaces of stable maps from smooth domain curves via the following procedure. Given a stable map $\left(C, x_{1}, x_{2}, f\right)$, consider the normalization of $C$. It consists of a disjoint union of smooth curves $C_{i}$ corresponding to the components of $C$, and there are maps $f_{i}$ from each curve to $\mathbb{P}^{r}$ naturally induced by $f$. Furthermore, auxiliary marked points are added to retain data about the node locations. The result is a collection of stable maps with smooth domain curves, one for each component of $C$. The evaluations of auxiliary marked points corresponding to the same node must agree. This gives rise to a fiber product of moduli spaces type $\mathcal{M}_{0, n}\left(\mathbb{P}^{r}, d\right)$, together with a morphism onto the stratum coming from the normalization map. There can be automorphisms of the stable maps in the stratum that are not accounted for by the fiber product. These occur when there is a collection of connected unions $U_{i}$ of components that satisfy the following conditions:

(1) none of the $U_{i}$ contain any marked points;

(2) restrictions of $f$ to $U_{i}$ and $U_{j}$ give isomorphic maps for all $i$ and $j$. 
These automorphisms correspond exactly to the automorphisms of the dual graph $\Gamma$ of the stratum. Proving the assertion that $\operatorname{Aut}(\Gamma)$ is the right group to quotient by appears quite complicated in general, but we can see it directly for the strata of $\overline{\mathcal{M}}_{0,2}\left(\mathbb{P}^{r}, 2\right)$. When stratified according to the dual graphs of stable maps, $\overline{\mathcal{M}}_{0,2}\left(\mathbb{P}^{r}, 2\right)$ has 9 types of strata. The corresponding dual graphs are shown below.
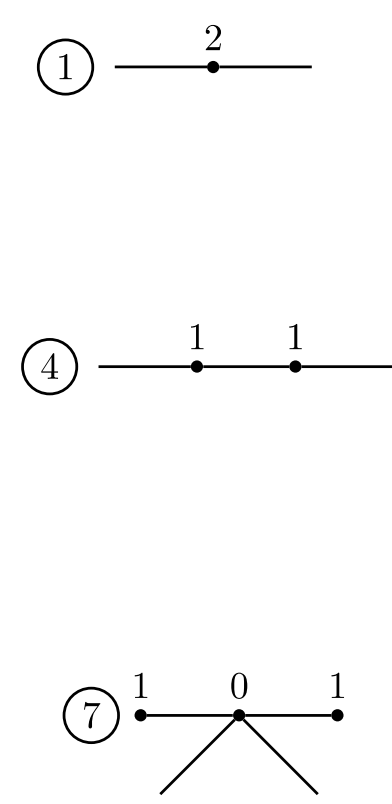

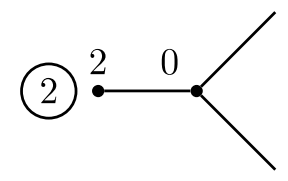

(5)
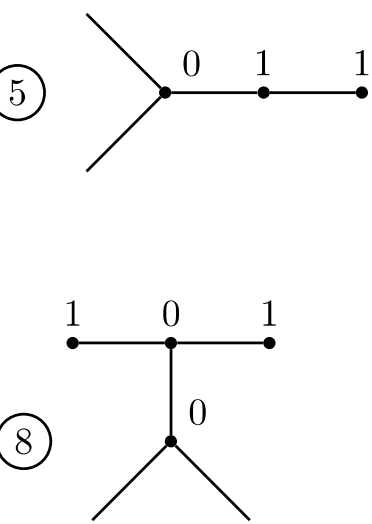
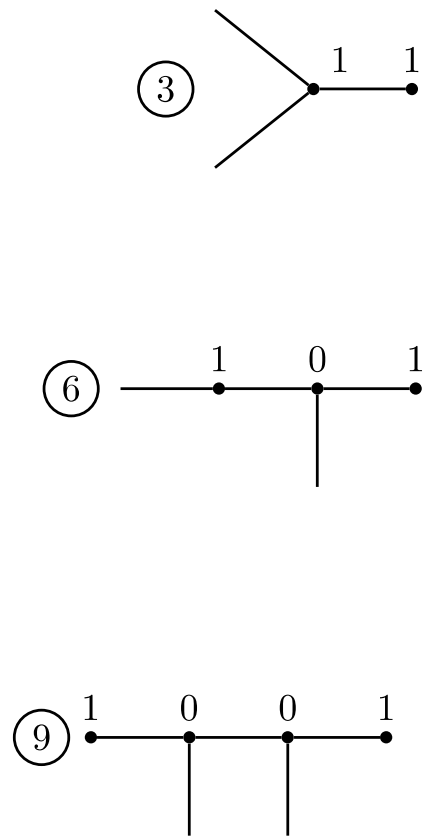

All the stratum types are listed, and the assertion clearly holds in each case. So we can compute the Serre polynomials of the strata using Corollary 1 and Proposition 3.

There are actually 10 strata, because there are two strata of type 6 depending on which marked point is identified with which tail on the graph. We use the same numbers to label the strata as those labeling the corresponding graphs above. Eight of the strata have no automorphisms, so we can directly compute ordinary Serre polynomials in these cases. The strata corresponding to Graphs 7 and 8 have automorphism group $S_{2}$. Calculating the $S_{2}$-equivariant Serre polynomials of these strata is necessary as an intermediate step. We now compute the Serre polynomials of the strata.

Stratum 1 is $\mathcal{M}_{0,2}\left(\mathbb{P}^{r}, 2\right)$. It is an $F\left(\mathbb{P}^{1}, 2\right)$-bundle over $\mathcal{M}_{0,0}\left(\mathbb{P}^{r}, 2\right)$. Thus Stratum 1 has Serre polynomial

$$
\operatorname{Serre}\left(F\left(\mathbb{P}^{1}, 2\right)\right) \operatorname{Serre}\left(\mathcal{M}_{0,0}\left(\mathbb{P}^{r}, 2\right)\right)=\left(q^{2}+q\right) q^{r+1} \frac{[r+1][r]}{[2]}=q^{r+2}[r+1][r] .
$$

Stratum 2 is isomorphic to the fiber product

$$
\mathcal{M}_{0,1}\left(\mathbb{P}^{r}, 2\right) \times_{\mathbb{P}^{r}} \mathcal{M}_{0,3}\left(\mathbb{P}^{r}, 0\right) .
$$

Now $\mathcal{M}_{0,3}\left(\mathbb{P}^{r}, 0\right) \simeq \mathbb{P}^{r}$, so the Serre polynomial of this stratum is just

$$
\operatorname{Serre}\left(\mathcal{M}_{0,1}\left(\mathbb{P}^{r}, 2\right)\right)=\operatorname{Serre}\left(\mathbb{P}^{1}\right) \operatorname{Serre}\left(\mathcal{M}_{0,0}\left(\mathbb{P}^{r}, 2\right)\right)=q^{r+1}[r+1][r]
$$

since $\mathcal{M}_{0,1}\left(\mathbb{P}^{r}, 2\right)$ is a $\mathbb{P}^{1}$-bundle over $\mathcal{M}_{0,0}\left(\mathbb{P}^{r}, 2\right)$.

Stratum 3 is isomorphic to the fiber product

$$
\mathcal{M}_{0,3}\left(\mathbb{P}^{r}, 1\right) \times_{\mathbb{P}^{r}} \mathcal{M}_{0,1}\left(\mathbb{P}^{r}, 1\right) .
$$

The $F\left(\mathbb{P}^{1}, 3\right)$-bundle $\mathcal{M}_{0,3}\left(\mathbb{P}^{r}, 1\right)$ over $\mathcal{M}_{0,0}\left(\mathbb{P}^{r}, 1\right)$ has Serre polynomial $\left(q^{3}-q\right)\left[\begin{array}{c}r+1 \\ 2\end{array}\right]$. Similarly, $\operatorname{Serre}\left(\mathcal{M}_{0,1}\left(\mathbb{P}^{r}, 1\right)\right)=(q+1)\left[\begin{array}{c}r+1 \\ 2\end{array}\right]=[r+1][r]$. Thus Stratum 3 has Serre polynomial

$$
\frac{\left(q^{3}-q\right)\left[\begin{array}{c}
r+1 \\
2
\end{array}\right][r+1][r]}{[r+1]}=\left(q^{2}-q\right)[r+1][r]^{2} .
$$


Stratum 4 is isomorphic to the fiber product

$$
\mathcal{M}_{0,2}\left(\mathbb{P}^{r}, 1\right) \times_{\mathbb{P}^{r}} \mathcal{M}_{0,2}\left(\mathbb{P}^{r}, 1\right) .
$$

The $F\left(\mathbb{P}^{1}, 2\right)$-bundle $\mathcal{M}_{0,2}\left(\mathbb{P}^{r}, 1\right)$ over $\mathcal{M}_{0,0}\left(\mathbb{P}^{r}, 1\right)$ has Serre polynomial $\left(q^{2}+q\right)\left[\begin{array}{c}r+1 \\ 2\end{array}\right]$. Thus Stratum 4 has Serre polynomial

$$
\frac{\left(q^{2}+q\right)^{2}[r+1]^{2}[r]^{2}}{[r+1][2]^{2}}=q^{2}[r+1][r]^{2} .
$$

Stratum 5 is isomorphic to the fiber product

$$
\mathcal{M}_{0,3}\left(\mathbb{P}^{r}, 0\right) \times_{\mathbb{P}^{r}} \mathcal{M}_{0,2}\left(\mathbb{P}^{r}, 1\right) \times \mathbb{P}^{r} \mathcal{M}_{0,1}\left(\mathbb{P}^{r}, 1\right),
$$

and this in turn is isomorphic to $\mathcal{M}_{0,2}\left(\mathbb{P}^{r}, 1\right) \times_{\mathbb{P}^{r}} \mathcal{M}_{0,1}\left(\mathbb{P}^{r}, 1\right)$. So Stratum 5 has Serre polynomial

$$
\frac{\left(q^{2}+q\right)\left[\begin{array}{c}
r+1 \\
2
\end{array}\right](q+1)\left[\begin{array}{c}
r+1 \\
2
\end{array}\right]}{[r+1]}=q[r+1][r]^{2} .
$$

A stratum of type 6 is isomorphic to the fiber product

$$
\mathcal{M}_{0,2}\left(\mathbb{P}^{r}, 1\right) \times_{\mathbb{P}^{r}} \mathcal{M}_{0,3}\left(\mathbb{P}^{r}, 0\right) \times_{\mathbb{P}^{r}} \mathcal{M}_{0,1}\left(\mathbb{P}^{r}, 1\right) .
$$

This is isomorphic to $\mathcal{M}_{0,2}\left(\mathbb{P}^{r}, 1\right) \times_{\mathbb{P}^{r}} \mathcal{M}_{0,1}\left(\mathbb{P}^{r}, 1\right)$, so each stratum of type 6 has Serre polynomial

$$
\frac{\left(q^{2}+q\right)\left[\begin{array}{c}
r+1 \\
2
\end{array}\right](q+1)\left[\begin{array}{c}
r+1 \\
2
\end{array}\right]}{[r+1]}=q[r+1][r]^{2} .
$$

Thus the total contribution from strata of type 6 is

$$
2 q[r+1][r]^{2} .
$$

Stratum 9 is isomorphic to the fiber product

$$
\mathcal{M}_{0,1}\left(\mathbb{P}^{r}, 1\right) \times_{\mathbb{P}^{r}} \mathcal{M}_{0,3}\left(\mathbb{P}^{r}, 0\right) \times_{\mathbb{P}^{r}} \mathcal{M}_{0,3}\left(\mathbb{P}^{r}, 0\right) \times_{\mathbb{P}^{r}} \mathcal{M}_{0,1}\left(\mathbb{P}^{r}, 1\right)
$$

It has Serre polynomial

$$
\frac{(q+1)^{2}\left[\begin{array}{c}
r+1 \\
2
\end{array}\right]^{2}}{[r+1]}=[r+1][r]^{2} .
$$

We now turn our attention to the two strata with automorphisms. Stratum 8 is isomorphic to the quotient of

$$
X=\mathcal{M}_{0,3}\left(\mathbb{P}^{r}, 0\right) \times_{\mathbb{P}^{r}} \mathcal{M}_{0,3}\left(\mathbb{P}^{r}, 0\right) \times_{\left(\mathbb{P}^{r}\right)^{2}} \mathcal{M}_{0,1}\left(\mathbb{P}^{r}, 1\right)^{2}
$$

by the action of $S_{2}$. The first copy of $\mathcal{M}_{0,3}\left(\mathbb{P}^{r}, 0\right)$ is superfluous. The action of $S_{2}$ on the cohomology of the second copy of $\mathcal{M}_{0,3}\left(\mathbb{P}^{r}, 0\right)$ is trivial. The action switches the two factors of $\mathcal{M}_{0,1}\left(\mathbb{P}^{r}, 1\right)$ as well as the two factors in $\mathbb{P}^{r} \times \mathbb{P}^{r}$. Since $\mathcal{M}_{0,1}\left(\mathbb{P}^{r}, 1\right)$ is a fiber space over $\mathbb{P}^{r}$, we can use Lemma 2 and Corollary 1 in computing the equivariant Serre polynomial of $X$ to be

$$
\begin{aligned}
\operatorname{Serre}_{2} & \left(\mathcal{M}_{0,3}\left(\mathbb{P}^{r}, 0\right)\right) \operatorname{Serre}_{2}\left(\left(\mathcal{M}_{0,1}\left(\mathbb{P}^{r}, 1\right) / \mathbb{P}^{r}\right)^{2}\right) \\
= & {[r+1]\left(\sigma_{2}\left(\frac{\operatorname{Serre}\left(\mathcal{M}_{0,1}\left(\mathbb{P}^{r}, 1\right)\right)}{\operatorname{Serre}\left(\mathbb{P}^{r}\right)}\right) \mathbb{1}+\lambda_{2}\left(\frac{\operatorname{Serre}\left(\mathcal{M}_{0,1}\left(\mathbb{P}^{r}, 1\right)\right)}{\operatorname{Serre}\left(\mathbb{P}^{r}\right)}\right) \epsilon\right) } \\
= & {[r+1]\left(\sigma_{2}([r]) \mathbb{1}+\lambda_{2}([r]) \epsilon\right) }
\end{aligned}
$$




$$
=[r+1]\left(\left[\begin{array}{c}
r+1 \\
2
\end{array}\right] \mathbb{1}+q\left[\begin{array}{l}
r \\
2
\end{array}\right] \epsilon\right) .
$$

(As in the proof of Lemma 1 , the fiber $\mathcal{M}_{0,1}\left(\mathbb{P}^{r}, 1\right) / \mathbb{P}^{r}$ is isomorphic to $\mathbb{P}^{r-1}$.) Now augmentation gives

$$
\frac{[r+1]^{2}[r]}{[2]}
$$

as the Serre polynomial of Stratum 8.

Stratum 7 is isomorphic to the quotient of

$$
Y=\mathcal{M}_{0,1}\left(\mathbb{P}^{r}, 1\right)^{2} \times{ }_{\left(\mathbb{P}^{r}\right)^{2}} \mathcal{M}_{0,4}\left(\mathbb{P}^{r}, 0\right)
$$

by the action of $S_{2}$, which again switches the squared factors. In addition, it switches two of the four marked points in $\mathcal{M}_{0,4}\left(\mathbb{P}^{r}, 0\right)$. Now $\mathcal{M}_{0,4}\left(\mathbb{P}^{r}, 0\right) \simeq M_{0,4} \times \mathbb{P}^{r}$, and $S_{2}$ acts trivially on the $\mathbb{P}^{r}$ factor. Furthermore, $M_{0,4} \simeq \mathbb{P}^{1} \backslash\{0,1, \infty\}$ has Serre polynomial $q-2$. But we need to know $\operatorname{Serre}_{2}\left(M_{0,4}\right)$ under an $S_{2}$-action switching two of the deleted points. It is not hard to imagine that $\operatorname{Serre}_{2}\left(M_{0,4}\right)=(q-1) \mathbb{1}-\epsilon$, but this takes some work to prove. Considering $M_{0,4}$ as the parameter space of four distinct points in $\mathbb{P}^{1}$ modulo automorphisms of $\mathbb{P}^{1}$, we obtain $M_{0,4} \simeq F\left(\mathbb{P}^{1}, 4\right) / \mathrm{PGL}(2)$. Now $\operatorname{PGL}(2)$ acts freely on $F\left(\mathbb{P}^{1}, 4\right)$. As a result,

$$
\operatorname{Serre}_{2}\left(M_{0,4}\right)=\frac{\operatorname{Serre}_{2}\left(F\left(\mathbb{P}^{1}, 4\right)\right)}{\operatorname{Serre}_{2}(\operatorname{PGL}(2))} .
$$

Since the cohomology of PGL(2) is not affected by the action,

$$
\text { Serre }_{2}(\operatorname{PGL}(2))=\operatorname{Serre}(\operatorname{PGL}(2))=q^{3}-q .
$$

We can stratify $\left(\mathbb{P}^{1}\right)^{4}$ into fifteen cells whose closures are respectively $\left(\mathbb{P}^{1}\right)^{4}$, the six large diagonals, the seven "medium diagonals" where two coordinate identifications are made, and the small diagonal, so that $F\left(\mathbb{P}^{1}, 4\right)$ is the complement of the union of all the cells corresponding to diagonals. We examine how the action affects cells of each type, subtracting the polynomials for cells that are removed. For concreteness, suppose the first two marked points are switched. Then two Chow classes in $A^{*}\left(\left(\mathbb{P}^{1}\right)^{4}\right)$ are switched if and only if their difference is a multiple of $H_{2}-H_{1}$ (where $H_{i}$ is the standard Chow generator of $A^{*}\left(\left(\mathbb{P}^{1}\right)^{4}\right)$ obtained by pulling back the hyperplane class of $\mathbb{P}^{1}$ under the $i$ 'th projection), so it is not hard to get

$$
\text { Serre }_{2}\left(\left(\mathbb{P}^{1}\right)^{4}\right)=\left(q^{4}+3 q^{3}+4 q^{2}+3 q+1\right) \mathbb{1}+\left(q^{3}+2 q^{2}+q\right) \epsilon .
$$

How does the action affect the diagonals removed from $\left(\mathbb{P}^{1}\right)^{4}$ ? Exactly two pairs, $\left(\Delta_{13}, \Delta_{23}\right)$ and $\left(\Delta_{14}, \Delta_{24}\right)$, of the six large diagonals are switched, so the corresponding cells contribute

$$
\left(-4 q^{3}+4 q\right) \mathbb{1}+\left(-2 q^{3}+2 q\right) \epsilon
$$

to the equivariant Serre polynomial, since these diagonals have been removed. Exactly two pairs, $\left(\Delta_{134}, \Delta_{234}\right)$ and $\left(\Delta_{(13)(24)}, \Delta_{(14)(23)}\right)$, among the seven diagonals with two identifications are switched as well. The corresponding cells contribute

$$
\left(-5 q^{2}-5 q\right) \mathbb{1}+\left(-2 q^{2}-2 q\right) \epsilon
$$

to the equivariant Serre polynomial. The small diagonal is not affected by the action, so it contributes

$$
(-q-1) \mathbb{1}
$$


Putting these together gives

$$
\operatorname{Serre}_{2}\left(F\left(\mathbb{P}^{1}, 4\right)\right)=\left(q^{4}-q^{3}-q^{2}+q\right) \mathbb{1}+\left(-q^{3}+q\right) \epsilon .
$$

Then by (3) and (4), we have the desired result $\operatorname{Serre}_{2}\left(M_{0,4}\right)=(q-1) \mathbb{1}-\epsilon$. Using Corollary 1 again, we thus calculate the equivariant Serre polynomial of $Y$ to be

$$
\begin{aligned}
& \operatorname{Serre}_{2}\left(\mathcal{M}_{0,4}\left(\mathbb{P}^{r}, 0\right)\right) \operatorname{Serre}_{2}\left(\left(\mathcal{M}_{0,1}\left(\mathbb{P}^{r}, 1\right) / \mathbb{P}^{r}\right)^{2}\right) \\
& \quad=[r+1]((q-1) \mathbb{1}-\epsilon)\left(\left[\begin{array}{c}
r+1 \\
2
\end{array}\right] \mathbb{1}+q\left[\begin{array}{l}
r \\
2
\end{array}\right] \epsilon\right) \\
& \quad=[r+1]\left(\left((q-1)\left[\begin{array}{c}
r+1 \\
2
\end{array}\right]-q\left[\begin{array}{l}
r \\
2
\end{array}\right]\right) \mathbb{1}+\left(\left(q^{2}-q\right)\left[\begin{array}{c}
r \\
2
\end{array}\right]-\left[\begin{array}{c}
r+1 \\
2
\end{array}\right]\right)\right) \epsilon .
\end{aligned}
$$

Augmentation gives

$$
\begin{array}{r}
{[r+1]\left((q-1)\left[\begin{array}{c}
r+1 \\
2
\end{array}\right]-q\left[\begin{array}{l}
r \\
2
\end{array}\right]\right)=\frac{[r+1][r]}{[2]}((q-1)[r+1]-q[r-1])} \\
=\frac{[r+1][r]}{[2]}\left(q^{r+1}+q^{r}\right)-\frac{[r+1]^{2}[r]}{[2]}=[r+1][r] q^{r}-\frac{[r+1]^{2}[r]}{[2]}
\end{array}
$$

as the Serre polynomial of Stratum 7 .

To get the Serre polynomial for the whole moduli space, we add together the contributions from all the strata

$$
\begin{aligned}
\text { Serre } & \left(\overline{\mathcal{M}}_{0,2}\left(\mathbb{P}^{r}, 2\right)\right) \\
= & q^{r+2}[r+1][r]+q^{r+1}[r+1][r]+\left(q^{2}-q\right)[r+1][r]^{2}+q^{2}[r+1][r]^{2}+q[r+1][r]^{2} \\
& +2 q[r+1][r]^{2}+[r+1][r]^{2}+\frac{[r+1]^{2}[r]}{[2]}+[r+1][r] q^{r}-\frac{[r+1]^{2}[r]}{[2]} \\
= & {[r+1][r]\left(q^{r+2}+q^{r+1}+\left(q^{2}-q\right)[r]+q^{2}[r]+3 q[r]+[r]+q^{r}\right) } \\
= & {[r+1][r]\left(q^{r+2}+q^{r+1}+q^{r}+[r]\left(2 q^{2}+2 q+1\right)\right) } \\
= & {[r+1][r]\left(q^{r+2}+q^{r+1}+q^{r}+2 \sum_{i=2}^{r+1} q^{i}+2 \sum_{i=1}^{r} q^{i}+\sum_{i=0}^{r-1} q^{i}\right) } \\
= & \left(\sum_{i=0}^{r} q^{i}\right)\left(\sum_{i=0}^{r-1} q^{i}\right)\left(\sum_{i=0}^{r+2} q^{i}+2 \sum_{i=1}^{r+1} q^{i}+2 \sum_{i=2}^{r} q^{i}\right) .
\end{aligned}
$$

Evaluating this sum at $q=1$ gives the Euler characteristic $(r+1) r(5 r+3)$.

\subsection{Formulas for the Betti numbers of $\overline{\mathcal{M}}_{0,2}\left(\mathbb{P}^{r}, 2\right)$}

Let $\alpha_{i}$ denote the $i$ 'th Betti number of the flag variety $\mathbb{F}(0,1 ; r)$ of point-line pairs in $\mathbb{P}^{r}$ such that the point lies on the line. Recall from the proof of Lemma 1 that $\operatorname{Serre}(\mathbb{F}(0,1 ; r))=[r+1][r]$. The product $[r+1][r]$ also appears as a factor in the Serre polynomial $(2)$ of $\overline{\mathcal{M}}_{0,2}\left(\mathbb{P}^{r}, 2\right)$, making its coefficients especially relevant to our computations. It is easy to see that the Betti numbers of $\mathbb{F}(0,1 ; r)$ initially follow the pattern $(1,2,3, \ldots)$, so that for the first half of the Betti numbers we have $\alpha_{i}=i+1$. Since $\operatorname{dim} \mathbb{F}(0,1 ; r)=2 r-1$ is always odd, it always has an even number of Betti numbers. By Poincaré duality, it follows that the middle two Betti numbers are both $r$, and the Betti numbers then decrease back to 1. It can be checked that all the Betti numbers are given by the formula

$$
\alpha_{i}=r+\frac{1}{2}-\left|r-\frac{1}{2}-i\right|
$$

for $i \in 0 \cup \underline{2 r-1}$, and $\alpha_{i}=0$ otherwise. 
Let $\beta_{j}$ be the $j$ 'th Betti number of $\overline{\mathcal{M}}_{0,2}\left(\mathbb{P}^{r}, 2\right)$. By distributing over the rightmost set of parentheses in Equation 2, we can reduce the computation of $\beta_{j}$ to finding coefficients of expressions of the form $[r+1][r][m]$, where $m \in\{r-1, r+1, r+3\}$. But these can be expressed in terms of the $\alpha_{i}$, and in this way we get the following formulas for the Betti numbers of $\overline{\mathcal{M}}_{0,2}\left(\mathbb{P}^{r}, 2\right)$ :

$$
\beta_{j}= \begin{cases}\sum_{i=0}^{j} \alpha_{i}+2 \sum_{i=0}^{j-1} \alpha_{i}+2 \sum_{i=0}^{j-2} \alpha_{i} & \text { if } j \leq r, \\ \sum_{i=0}^{r+1} \alpha_{i}+2 \sum_{i=0}^{r} \alpha_{i}+2 \sum_{i=1}^{r-1} \alpha_{i} & \text { if } j=r+1, \\ \sum_{i=j-r-2}^{j} \alpha_{i}+2 \sum_{i=j-r-1}^{j-1} \alpha_{i}+2 \sum_{i=j-r}^{j-2} \alpha_{i} & \text { if } r+2 \leq j \leq 2 r-1, \\ \sum_{i=r-2}^{2 r-1} \alpha_{i}+2 \sum_{i=r-1}^{2 r-1} \alpha_{i}+2 \sum_{i=r}^{2 r-2} \alpha_{i} & \text { if } j=2 r \\ \sum_{i=j-r-2}^{2 r-1} \alpha_{i}+2 \sum_{i=j-r-1}^{2 r-1} \alpha_{i}+2 \sum_{i=j-r}^{2 r-1} \alpha_{i} & \text { if } 2 r+1 \leq j \leq 3 r+1 .\end{cases}
$$

We can come up with an especially explicit description of $\beta_{j}$ for $j<r$ since we know $\alpha_{i}=i+1$ for $i<r$. Also, $\alpha_{r}=r$, which gives the second part below.

Corollary 2. 1. For $j<r$, the $j$ 'th Betti number of $\overline{\mathcal{M}}_{0,2}\left(\mathbb{P}^{r}, 2\right)$ is

$$
\beta_{j}=\frac{5}{2} j^{2}+\frac{3}{2} j+1
$$

2. Furthermore

$$
\beta_{r}=\frac{5}{2} r^{2}+\frac{3}{2} r .
$$

As a consequence of this, a particular Betti number of $\overline{\mathcal{M}}_{0,2}\left(\mathbb{P}^{r}, 2\right)$ stabilizes as $r$ becomes large.

Corollary 3. For all $r>j$, the $j$ 'th Betti number of $\overline{\mathcal{M}}_{0,2}\left(\mathbb{P}^{r}, 2\right)$ is $\beta_{j}=\frac{5}{2} j^{2}+\frac{3}{2} j+1$.

Let $\bar{\beta}_{j}$ be this limiting value. We have

$$
\bar{\beta}_{0}=1, \quad \bar{\beta}_{1}=5, \quad \bar{\beta}_{2}=14, \quad \bar{\beta}_{3}=28, \quad \bar{\beta}_{4}=47, \quad \bar{\beta}_{5}=71, \quad \ldots
$$

\subsection{Poincaré polynomials of $\overline{\mathcal{M}}_{0,1}\left(\mathbb{P}^{r}, 2\right)$ and $\overline{\mathcal{M}}_{0,2}\left(\mathbb{P}^{r}, 2\right)$ for small $r$}

Using the same procedure as above, one can easily compute the Poincaré polynomial of $\overline{\mathcal{M}}_{0,1}\left(\mathbb{P}^{r}, 2\right)$, which is also needed in the sequel [4].

Proposition 5. If $r$ is even, the Poincaré polynomial of $\overline{\mathcal{M}}_{0,1}\left(\mathbb{P}^{r}, 2\right)$ is

$$
\operatorname{Serre}\left(\overline{\mathcal{M}}_{0,1}\left(\mathbb{P}^{r}, 2\right)\right)=\left(\sum_{i=0}^{r} q^{i}\right)\left(\sum_{i=0}^{(r-2) / 2} q^{2 i}\right)\left(\sum_{i=0}^{r+2} q^{i}+\sum_{i=1}^{r+1} q^{i}+\sum_{i=2}^{r} q^{i}\right),
$$

and if $r$ is odd, the Poincaré polynomial of $\overline{\mathcal{M}}_{0,1}\left(\mathbb{P}^{r}, 2\right)$ is

$$
\operatorname{Serre}\left(\overline{\mathcal{M}}_{0,1}\left(\mathbb{P}^{r}, 2\right)\right)=\left(\sum_{i=0}^{r-1} q^{i}\right)\left(\sum_{i=0}^{(r-1) / 2} q^{2 i}\right)\left(\sum_{i=0}^{r+2} q^{i}+\sum_{i=1}^{r+1} q^{i}+\sum_{i=2}^{r} q^{i}\right) .
$$

Thus, for small values of $r$, we get the explicit Poincaré polynomials listed in Tables 1 and 2 . 
Table 1. Euler characteristics and Poincaré polynomials for $X=\overline{\mathcal{M}}_{0,1}\left(\mathbb{P}^{r}, 2\right)$.

\begin{tabular}{|c|c|c|}
\hline$r$ & $\overline{\chi(X)}$ & $\operatorname{Serre}(X)$ \\
\hline 1 & 6 & $1+2 q+2 q^{2}+q^{3}$ \\
\hline 2 & 27 & $1+3 q+6 q^{2}+7 q^{3}+6 q^{4}+3 q^{5}+q^{6}$ \\
\hline 3 & 72 & $1+3 q+7 q^{2}+11 q^{3}+14 q^{4}+14 q^{5}+11 q^{6}+7 q^{7}+3 q^{8}+q^{9}$ \\
\hline 4 & 150 & $\begin{array}{l}1+3 q+7 q^{2}+12 q^{3}+18 q^{4}+22 q^{5}+24 q^{6} \\
\quad+22 q^{7}+18 q^{8}+12 q^{9}+7 q^{10}+3 q^{11}+q^{12}\end{array}$ \\
\hline 5 & 270 & $\begin{array}{l}1+3 q+7 q^{2}+12 q^{3}+19 q^{4}+26 q^{5}+32 q^{6}+35 q^{7} \\
\quad+35 q^{8}+32 q^{9}+26 q^{10}+19 q^{11}+12 q^{12}+7 q^{13}+3 q^{14}+q^{15}\end{array}$ \\
\hline 6 & 441 & $\begin{array}{l}1+3 q+7 q^{2}+12 q^{3}+19 q^{4}+27 q^{5}+36 q^{6}+43 q^{7}+48 q^{8}+49 q^{9} \\
\quad+48 q^{10}+43 q^{11}+36 q^{12}+27 q^{13}+19 q^{14}+12 q^{15}+7 q^{16}+3 q^{17}+q^{18}\end{array}$ \\
\hline 7 & 672 & $\begin{array}{l}1+3 q+7 q^{2}+12 q^{3}+19 q^{4}+27 q^{5}+37 q^{6}+47 q^{7} \\
\quad+56 q^{8}+62 q^{9}+65 q^{10}+65 q^{11}+62 q^{12}+56 q^{13}+47 q^{14} \\
\quad+37 q^{15}+27 q^{16}+19 q^{17}+12 q^{18}+7 q^{19}+3 q^{20}+q^{21}\end{array}$ \\
\hline
\end{tabular}

Table 2. Euler characteristics and Poincaré polynomials for $Y=\overline{\mathcal{M}}_{0,2}\left(\mathbb{P}^{r}, 2\right)$.

\begin{tabular}{|c|c|c|}
\hline & $\chi(Y)$ & Serre $(Y)$ \\
\hline 1 & 16 & $1+4 q+6 q^{2}+4 q^{3}+q^{4}$ \\
\hline 2 & 78 & $1+5 q+13 q^{2}+20 q^{3}+20 q^{4}+13 q^{5}+5 q^{6}+q^{7}$ \\
\hline 3 & 216 & $1+5 q+14 q^{2}+27 q^{3}+39 q^{4}+44 q^{5}+39 q^{6}+27 q^{7}+14 q^{8}+5 q^{9}+q^{10}$ \\
\hline 4 & 460 & $\begin{array}{l}1+5 q+14 q^{2}+28 q^{3}+46 q^{4}+63 q^{5}+73 q^{6}+73 q^{7} \\
\quad+63 q^{8}+46 q^{9}+28 q^{10}+14 q^{11}+5 q^{12}+q^{13}\end{array}$ \\
\hline 5 & 840 & $\begin{array}{l}1+5 q+14 q^{2}+28 q^{3}+47 q^{4}+70 q^{5}+92 q^{6}+107 q^{7}+112 q^{8} \\
\quad+107 q^{9}+92 q^{10}+70 q^{11}+47 q^{12}+28 q^{13}+14 q^{14}+5 q^{15}+q^{16}\end{array}$ \\
\hline 6 & 1386 & $\begin{array}{l}1+5 q+14 q^{2}+28 q^{3}+47 q^{4}+71 q^{5}+99 q^{6}+126 q^{7} \\
\quad+146 q^{8}+156 q^{9}+156 q^{10}+146 q^{11}+126 q^{12}+99 q^{13} \\
\quad+71 q^{14}+47 q^{15}+28 q^{16}+14 q^{17}+5 q^{18}+q^{19}\end{array}$ \\
\hline 7 & 2128 & $\begin{array}{l}1+5 q+14 q^{2}+28 q^{3}+47 q^{4}+71 q^{5}+100 q^{6}+133 q^{7}+165 q^{8} \\
\quad+190 q^{9}+205 q^{10}+210 q^{11}+205 q^{12}+190 q^{13}+165 q^{14}+133 q^{15} \\
\quad+100 q^{16}+71 q^{17}+47 q^{18}+28 q^{19}+14 q^{20}+5 q^{21}+q^{22}\end{array}$ \\
\hline
\end{tabular}

\section{An additive basis for $\overline{\mathcal{M}}_{0,2}\left(\mathbb{P}^{r}, 2\right)$}

A presentation for the Chow rings $A^{*}\left(\mathcal{M}_{0,0}\left(\mathbb{P}^{r}, d\right)\right)$ is described in [21] (see also [2]). Naturally then, an additive basis for these rings is readily available. In this section, we describe an additive basis for $A^{*}\left(\overline{\mathcal{M}}_{0,2}\left(\mathbb{P}^{r}, 2\right)\right)$ in terms of the additive bases for $A^{*}\left(\mathcal{M}_{0,0}\left(\mathbb{P}^{r}, 1\right)\right)$ and $A^{*}\left(\mathcal{M}_{0,0}\left(\mathbb{P}^{r}, 2\right)\right)$ using a decomposition of $\overline{\mathcal{M}}_{0,2}\left(\mathbb{P}^{r}, 2\right)$ and excision. (Note that an additive basis has now been more explicitly described in [18].)

Let $X$ be the locus in $\overline{\mathcal{M}}_{0,2}\left(\mathbb{P}^{r}, 2\right)$ where the curve has two degree one components (with degree zero components also allowed). It is a divisor, and its complement is the open locus $U$ where the curve has a degree two component. In the notation of Section $2, U$ is the union of Strata 1 and 2.

Note that $U$ is a $\mathbb{P}^{1} \times \mathbb{P}^{1}$-bundle over $\mathcal{M}_{0,0}\left(\mathbb{P}^{r}, 2\right)$. Let $H_{1}$ and $H_{2}$ be the two hyperplane divisor classes in $\mathbb{P}^{1} \times \mathbb{P}^{1}$. Then any class in $A^{k}(U)$ can be expressed in the form $\alpha+\beta_{1} H_{1}+\beta_{2} H_{2}+\gamma H_{1} H_{2}$, where $\alpha \in A^{k}\left(\mathcal{M}_{0,0}\left(\mathbb{P}^{r}, 2\right)\right), \beta_{i} \in A^{k-1}\left(\mathcal{M}_{0,0}\left(\mathbb{P}^{r}, 2\right)\right)$, and $\gamma \in A^{k-2}\left(\mathcal{M}_{0,0}\left(\mathbb{P}^{r}, 2\right)\right)$. Thus we can write

$$
A^{k}(U) \simeq A^{k}\left(\mathcal{M}_{0,0}\left(\mathbb{P}^{r}, 2\right)\right) \oplus A^{k-1}\left(\mathcal{M}_{0,0}\left(\mathbb{P}^{r}, 2\right)\right) \oplus A^{k-1}\left(\mathcal{M}_{0,0}\left(\mathbb{P}^{r}, 2\right)\right) \oplus A^{k-2}\left(\mathcal{M}_{0,0}\left(\mathbb{P}^{r}, 2\right)\right)
$$


Consider the exact sequence

$$
A^{k-1}(X) \longrightarrow A^{k}\left(\overline{\mathcal{M}}_{0,2}\left(\mathbb{P}^{r}, 2\right)\right) \longrightarrow A^{k}(U) \longrightarrow 0 .
$$

This says $A^{k}\left(\overline{\mathcal{M}}_{0,2}\left(\mathbb{P}^{r}, 2\right)\right)$ is the direct sum of $A^{k}(U)$ and the image of $A^{k-1}(X)$. We have reduced to studying the latter. Let $Y$ be the sublocus of $X$ where there are no marked points on the degree one components. Then $Y$ is a divisor in $X$, and we can consider the exact sequence

$$
A^{k-2}(Y) \longrightarrow A^{k-1}(X) \longrightarrow A^{k-1}(V) \longrightarrow 0,
$$

where $V=X-Y$. We can further decompose $V$ into $V_{1} \amalg V_{2}$, where $V_{2}$ contains the locus where each degree one component has a marked point. In $V_{2}$ we also allow the second marked point to approach the node. In the notation of Section $2, V_{2}$ is the union of Stratum 4 and one of the strata of type 6 . Thus $V_{2}$ is a $\mathbb{P}^{1} \times \mathbb{A}^{1}$-bundle over the boundary divisor $D \simeq \overline{\mathcal{M}}_{0,1}\left(\mathbb{P}^{r}, 1\right) \times_{\mathbb{P}^{r}} \overline{\mathcal{M}}_{0,1}\left(\mathbb{P}^{r}, 1\right)$ in $\overline{\mathcal{M}}_{0,0}\left(\mathbb{P}^{r}, 2\right)$. Since the fiber product is a $\mathbb{P}^{r-1}$-bundle over $\overline{\mathcal{M}}_{0,1}\left(\mathbb{P}^{r}, 1\right)$, and $\overline{\mathcal{M}}_{0,1}\left(\mathbb{P}^{r}, 1\right)$ is a $\mathbb{P}^{1}$-bundle over $\overline{\mathcal{M}}_{0,0}\left(\mathbb{P}^{r}, 1\right) \simeq \mathcal{M}_{0,0}\left(\mathbb{P}^{r}, 1\right)$, we find overall that $D$ is a $\mathbb{P}^{r-1} \times \mathbb{P}^{1}$-bundle over $\mathcal{M}_{0,0}\left(\mathbb{P}^{r}, 1\right)$. Similarly, $V_{1}$, which is the union of Stratum 3, Stratum 5, and the other stratum of type 6 , is also a $\mathbb{P}^{1} \times \mathbb{A}^{1}$-bundle over $D$, and thus a $\mathbb{P}^{r-1} \times\left(\mathbb{P}^{1}\right)^{2} \times \mathbb{A}^{1}$-bundle over $\mathcal{M}_{0,0}\left(\mathbb{P}^{r}, 1\right)$. We have

$$
\begin{aligned}
A^{k-1}\left(V_{2}\right) \simeq A^{k-1}\left(V_{1}\right) \simeq & \oplus_{i=0}^{r-1}\left(A^{k-i-1}\left(\mathcal{M}_{0,0}\left(\mathbb{P}^{r}, 1\right)\right) \oplus A^{k-i-2}\left(\mathcal{M}_{0,0}\left(\mathbb{P}^{r}, 1\right)\right)\right. \\
& \left.\oplus A^{k-i-2}\left(\mathcal{M}_{0,0}\left(\mathbb{P}^{r}, 1\right)\right) \oplus A^{k-i-3}\left(\mathcal{M}_{0,0}\left(\mathbb{P}^{r}, 1\right)\right)\right) .
\end{aligned}
$$

Finally, $Y$ is the union of Strata 7,8 , and 9 , and is a $\mathbb{P}^{1}$-bundle over the codimension two boundary stratum $Z$ in $\overline{\mathcal{M}}_{0,1}\left(\mathbb{P}^{r}, 2\right)$. (This is the boundary locus where the domain curves have three components.) Now $Z$ is isomorphic to the $S_{2}$-quotient of $\overline{\mathcal{M}}_{0,1}\left(\mathbb{P}^{r}, 1\right)^{2} \times_{\left(\mathbb{P}^{r}\right)^{2}} \overline{\mathcal{M}}_{0,3}\left(\mathbb{P}^{r}, 0\right)$ that arises by switching the factors in the squares. This fiber product is isomorphic to a $\left(\mathbb{P}^{r-1}\right)^{2}-$ bundle over $\mathbb{P}^{r}$. So

$$
A^{k-2}(Y) \simeq\left(\oplus_{i=0}^{r-1} \oplus_{j=0}^{r-1} A^{k-i-j-2}\left(\mathbb{P}^{r}\right)\right)^{S_{2}} \oplus\left(\oplus_{i=0}^{r-1} \oplus_{j=0}^{r-1} A^{k-i-j-3}\left(\mathbb{P}^{r}\right)\right)^{S_{2}}
$$

Putting all this together, we attain an additive basis for $A^{*}\left(\overline{\mathcal{M}}_{0,2}\left(\mathbb{P}^{r}, 2\right)\right)$. It is given in degree $k$ by

$$
\begin{aligned}
A^{k}\left(\overline{\mathcal{M}}_{0,2}\left(\mathbb{P}^{r}, 2\right)\right) \simeq & A^{k}\left(\mathcal{M}_{0,0}\left(\mathbb{P}^{r}, 2\right)\right) \oplus A^{k-1}\left(\mathcal{M}_{0,0}\left(\mathbb{P}^{r}, 2\right)\right) \\
& \oplus A^{k-1}\left(\mathcal{M}_{0,0}\left(\mathbb{P}^{r}, 2\right)\right) \oplus A^{k-2}\left(\mathcal{M}_{0,0}\left(\mathbb{P}^{r}, 2\right)\right) \\
& \oplus \oplus_{i=0}^{r-1}\left(A^{k-i-1}\left(\mathcal{M}_{0,0}\left(\mathbb{P}^{r}, 1\right)\right) \oplus A^{k-i-2}\left(\mathcal{M}_{0,0}\left(\mathbb{P}^{r}, 1\right)\right)\right. \\
& \left.\oplus A^{k-i-2}\left(\mathcal{M}_{0,0}\left(\mathbb{P}^{r}, 1\right)\right) \oplus A^{k-i-3}\left(\mathcal{M}_{0,0}\left(\mathbb{P}^{r}, 1\right)\right)\right) \\
& \oplus \oplus_{i=0}^{r-1}\left(A^{k-i-1}\left(\mathcal{M}_{0,0}\left(\mathbb{P}^{r}, 1\right)\right) \oplus A^{k-i-2}\left(\mathcal{M}_{0,0}\left(\mathbb{P}^{r}, 1\right)\right)\right. \\
& \left.\oplus A^{k-i-2}\left(\mathcal{M}_{0,0}\left(\mathbb{P}^{r}, 1\right)\right) \oplus A^{k-i-3}\left(\mathcal{M}_{0,0}\left(\mathbb{P}^{r}, 1\right)\right)\right) \\
& \oplus\left(\oplus_{i=0}^{r-1} \oplus_{j=0}^{r-1} A^{k-i-j-2}\left(\mathbb{P}^{r}\right)\right)^{S_{2}} \oplus\left(\oplus_{i=0}^{r-1} \oplus_{j=0}^{r-1} A^{k-i-j-3}\left(\mathbb{P}^{r}\right)\right)^{S_{2}} .
\end{aligned}
$$

Of course, this is only a generating set a priori, but by comparing with the Betti numbers we can see that the generators are independent. In other words, we need only verify that the expression above gives the same Serre polynomial for $A^{*}\left(\overline{\mathcal{M}}_{0,2}\left(\mathbb{P}^{r}, 2\right)\right)$ as found in Section 2. 
Keeping in mind that the Serre polynomial grades by dimension rather than codimension, and using the notation of Section 2, from the decomposition above we obtain

$$
\begin{aligned}
(q+1)^{2} q^{r+1}\left[\begin{array}{c}
r+1 \\
2
\end{array}\right]+2 \sum_{i=0}^{r-1} q^{i+1}(q+1)^{2}\left[\begin{array}{c}
r+1 \\
2
\end{array}\right]+(q+1)[r+1] \sigma_{2}([r]) \\
=[r+1][r]\left((q+1) q^{r+1}+2 q[r](q+1)+[r+1]\right) \\
\quad=[r+1][r]\left(q^{r+2}+q^{r+1}+2 \sum_{i=1}^{r} q^{i}+2 \sum_{i=2}^{r+1} q^{i}+\sum_{i=0}^{r} q^{i}\right) \\
\quad=[r+1][r]\left(\sum_{i=0}^{r+2} q^{i}+2 \sum_{i=1}^{r+1} q^{i}+2 \sum_{i=2}^{r} q^{i}\right)
\end{aligned}
$$

in agreement with equation (2).

\section{Acknowledgements}

The content of this article derives from a part of my doctoral dissertation at Oklahoma State University. I am deeply grateful to my dissertation adviser, Sheldon Katz for financial support, insight, encouragement, and inspiration. William Jaco and Alan Adolphson provided additional funding during work on this project. I appreciate the hospitality of the University of Illinois mathematics department during my years as a visiting graduate student there. I also acknowledge with gratitude the Oklahoma State University mathematics department for extended support during that time.

\section{References}

[1] Behrend K., Manin Yu., Stacks of stable maps and Gromov-Witten invariants, Duke Math. J. 85 (1996), 1-60, alg-geom/9506023.

[2] Behrend K., O'Halloran A., On the cohomology of stable map spaces, Invent. Math. 154 (2003), 385-450, math.AG/0202288.

[3] Cox D.A., Katz S., Mirror symmetry and algebraic geometry, Mathematical Surveys and Monographs, Vol. 68, American Mathematical Society, Providence, Rhode Island, 1999.

[4] Cox J.A., A presentation for the Chow ring of $\overline{\mathcal{M}}_{0,2}\left(\mathbb{P}^{1}, 2\right)$, Comm. Algebra, to appear, math.AG/0504575.

[5] Danilov V.I., Khovanskiı̌A.G., Newton polyhedra and an algorithm for calculating Hodge-Deligne numbers, Math. USSR-Izv. 29 (1987), no. 2, 279-298.

[6] Deligne P., Théorie de Hodge, II, Inst. Hautes Études Sci. Publ. Math. 40 (1971), 5-57.

[7] Fulton W., MacPherson R., A compactification of configuration spaces, Ann. of Math. (2) 139 (1994), 183-225.

[8] Getzler E., Mixed Hodge structures of configuration spaces, Preprint 96-61, Max-Planck-Institut für Mathematik, Bonn, alg-geom/9510018.

[9] Getzler E., Pandharipande R., The Poincaré polynomial of $\bar{M}_{0, n}\left(\mathbb{P}^{r}, d\right)$, unpublished.

[10] Getzler E., Pandharipande R., The Betti numbers of $\overline{\mathcal{M}}_{0, n}(r, d)$, J. Algebraic Geom. 15 (2006), 709-732, math.AG/0502525.

[11] Keel S., Intersection theory of moduli space of stable $n$-pointed curves of genus zero, Trans. Amer. Math. Soc. 330 (1992), 545-574.

[12] Knutson D., $\lambda$-rings and the representation theory of the symmetric group, Lecture Notes in Mathematics, Vol. 308, Springer-Verlag, Berlin - New York, 1973.

[13] Kontsevich M., Enumeration of rational curves via torus actions, in The Moduli Space of Curves (Texel Island, 1994), Birkhäuser, Boston, 1995, 335-368, hep-th/9405035. 
[14] Kontsevich M., Manin Yu., Gromov-Witten classes, quantum cohomology, and enumerative geometry, Comm. Math. Phys. 164 (1994), 525-562, hep-th/9402147.

[15] Kontsevich M., Manin Yu., Relations between the correlators of the topological sigma-model coupled to gravity, Comm. Math. Phys. 196 (1998), 385-398, alg-geom/9708024.

[16] Macdonald I.G., Symmetric functions and Hall polynomials, Clarendon Press, Oxford, 1979.

[17] Mustaţă A., Mustaţă M.A., Intermediate moduli spaces of stable maps, Invent. Math. 167 (2007), 47-90, math.AG/0409569.

[18] Mustaţă A., Mustaţă M.A., The Chow ring of $\bar{M}_{0, m}(n, d)$, J. Reine Angew. Math., to appear, math.AG/0507464.

[19] Oprea D., Tautological classes on the moduli spaces of stable maps to $\mathbb{P}^{r}$ via torus actions, Adv. Math. 207 (2006), 661-690, math.AG/0404284.

[20] Oprea D., The tautological rings of the moduli spaces of stable maps to flag varieties, J. Algebraic Geom. 15 (2006), 623-655, math.AG/0404280.

[21] Pandharipande R., The Chow ring of the nonlinear Grassmannian, J. Algebraic Geom. 7 (1998), 123-140, alg-geom/9604022.

[22] Smith L., Homological algebra and the Eilenberg-Moore spectral sequence, Trans. Amer. Math. Soc. 129 (1967), 58-93. 